\title{
Dynamics of Methionine Ligand Rebinding in Cytochrome $\mathrm{c}$
}

\author{
Ping Zhang, Edyta Małolepsza, and John E. Straub* \\ Department of Chemistry, Boston University, Boston, Massachusetts 02215, United States
}

ABSTRACT: Geminate recombination of the methionine ligand to the heme iron in ferrous cytochrome $\mathrm{c}$ protein following photodissociation displays rich kinetics. It is of particular interest to develop an understanding of fast and slow rebinding time scales, observed in experimental studies, in terms of features of the underlying complex energy landscape. The classical empirical force field in the heme pocket has been extended by incorporating $a b$ initio potential energy surface calculations representing the ground singlet state and quintet state associated with methionine bond breaking and rebinding. An algorithm based on the Landau-Zener nonadiabatic transition theory has been employed to model the electronic surface hopping between two spin states during the process of ligand dissociation and recombination. Multiple conformational substates of the dissociated methionine ligand are found to participate in the reaction dynamics. Varying time scales for interconversion between substates lead to a mechanism elucidating the fast and slow rebinding time scales. The reaction system may be understood in terms of a two-dimensional reaction coordinate distinctly separated from the coupled bath of surrounding protein and solvent degrees of freedom. Insights into the reaction dynamics provided by this study lead to suggestions for future experiments to further probe the role of dynamic heterogeneity in the kinetics of ligand-protein binding.

\section{INTRODUCTION}

Geminate recombination of axial ligands to the iron atom in heme proteins, as one of the key steps of protein global structural dynamics and allostery, has been of great interest and widely studied both experimentally and theoretically in the past decades. Many of those studies have been dedicated to diatomic axial ligands, such as $\mathrm{CO}, \mathrm{NO}$, and $\mathrm{O}_{2}$ in globins. ${ }^{1-27}$ From those studies, a deep and general understanding of protein dynamics, in terms of transitions between conformational substates, has developed and formed the foundation for modern "energy landscape" theories of protein dynamics and thermodynamics. In spite of this success, a rigorous understanding of molecular reaction dynamics for ligand rebinding in myoglobin has proven elusive. The Agmon-Hopfield ${ }^{8}$ and Champion-Srajer ${ }^{28,29}$ models of ligand rebinding define specific reaction coordinates for the rebinding process but also include an undefined protein coordinate as an essential component of the multidimensional reaction coordinate in addition to the surrounding bath. Although heme-ligand dissociation in heme proteins usually does not occur under normal conditions, heme-ligand bond breaking and rebinding take place during the process of internal and external ligand switching or competition in neuroglobin $^{30,31}$ as well as in a specific group of heme-based sensor proteins such as Ec DOS, DOSH, and CooA. ${ }^{7,32,33}$ Understanding the kinetics and thermodynamics of internal ligand binding is essential to our knowledge of the composition and function of these heme proteins.

Cytochrome c (cyt c) (Figure 1) is an essential component of the electron transport chain in mitochondria. ${ }^{34}$ The 6coordinated iron $(\mathrm{Fe})$ in the heme plays an essential role in the process of electron transport. The dissociation of the internal
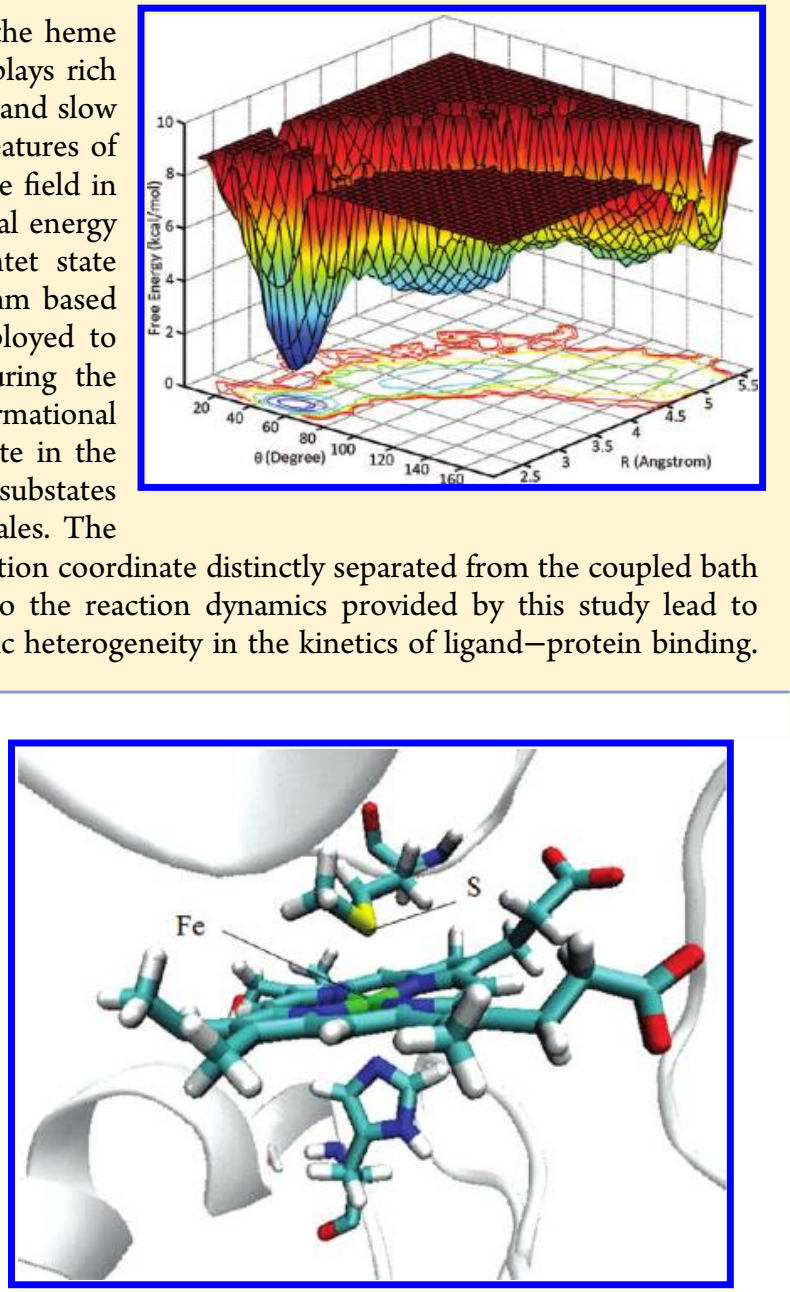

Figure 1. Heme pocket in cyt c showing distal Met80 and proximal His 18.

ligand, the methionine (Met80), from the heme causes an electronic structural change around the heme inducing the transition between 6-coordinated and 5-coordinated iron. Photodissociation of Met80 in reduced Fe(II) cyt $\mathrm{c}$ has been performed by Champion and co-workers, ${ }^{35}$ and the rebinding of the Met80 was observed to occur with single exponential kinetics on a time scale of 6.2 ps. Kruglik and co-workers ${ }^{36}$ studied the same system and two rebinding time scales were

Special Issue: B: Harold A. Scheraga Festschrift

Received: January 24, 2012

Revised: $\quad$ March 20, 2012

Published: March 20, 2012 
observed, including a fast and a slow rebinding with 4.8 ps and 16 ps time constant, respectively. In recent work using femtosecond transient-absorption method, Zhong and coworkers $^{37}$ observed a Met80 rebinding time constant of approximately $7 \mathrm{ps}$. In that study, the global structural distortion and energy relaxation triggered by the photodissociation was also observed. These studies raise questions regarding time scale mechanisms of fast and slow rebinding of Met80 in cyt c.

There are two main challenges that face the computational modeling of the Met80 rebinding in cyt c. (i) Identifying an effective potential energy surface (PES) that allows for ligand dissociation and recombination in a classical molecular dynamics (MD) simulation. (ii) Defining a reactive model to describe the electronic structural change, including changes in spin state, associated with the transition between bonded and dissociated ligand states.

In forming PESs for the rebinding process, one approach is to reduce the system to one or two degrees of freedom defining a one- or two-dimensional PES. The PES can be chosen from empirical classical MD force field or experimental data, or it can be obtained from ab initio calculations. A ligand-porphyrinimidazole model is often employed using geometrical optimizations and single point energy surface scans along a particular reaction coordinate. ${ }^{19-21}$ In a recent study of NO rebinding in myoglobin by Nutt, Karplus, and Meuwly, ${ }^{9}$ a twodimensional PES was developed using ab initio optimization on a NO-heme-imidazole system. PES calculations in different spin states have been intensely studied by Harvey and coworkers, ${ }^{21,38-40}$ with a focus on analyzing spin-forbidden nonadiabatic transitions. One problem associated with using a simplified model system to obtain the PES is that the effects of the protein environment are largely neglected. ${ }^{1,8,41-45}$

In defining the process of nonadiabatic transitions between PESs, various models and theories have been developed from studies of NO rebinding in myoglobin. ${ }^{9,11,13,15-17}$ A simple PES can be defined dependent on key heme-ligand atoms around the reaction center. $\mathrm{Li}$, Elber, and Straub ${ }^{17}$ employed the onedimensional Landau-Zener ${ }^{46-48}$ model to capture the nonadiabatic transitions between the bonded state and the dissociated state in NO binding in myoglobin. Two onedimensional PESs for two electronic states were designed, and the nonadiabatic transition associated with $\mathrm{NO}-$ heme bond breaking and rebinding was modeled using the Landau-Zener theory. The problem that arises is that the Landau-Zener theory, a powerful tool to investigate nonadiabatic transition for one-dimensional systems, is challenged to describe reaction dynamics in proteins where the multidimensional reaction degrees of freedom are essential, and the system cannot be reliably reduced to one or two dimensions. ${ }^{8,49}$ Meuwly, Karplus, and co-workers ${ }^{11}$ chose a different reactive model to describe the ligand rebinding process. A structural criterion for the reaction was defined, and once the system meets the criterion for a transition, the transition is assumed to always occur. The reaction rate is estimated from the energy barrier at the transition point of the PES. This method efficiently defines a reactant and a product state, but leads to an estimation of the reaction rate that ignores nonadiabatic tunneling transitions and barrier recrossing. ${ }^{48,50,51}$

Calculation of the nonadiabatic transition probability using the Landau-Zener theory requires knowledge of the coupling between two electronic states ${ }^{44,52-54}$, which is often difficult to determine. The application of the surface-hopping algorithm based on Tully's fewest switches model ${ }^{55,56}$, which demands a model for the nonadiabatic coupling vector, is a great challenge in complex systems such as ligand-heme binding in proteins.

We present a computational study of geminate recombination of the Met80 ligand to the heme iron atom in ferrous cyt $c$ protein following photodissociation in terms of the dynamics of the underlying complex energy landscape. The CHARMM force field is extended by incorporating $a b$ initio PES calculations to model the singlet (bonded) and quintet (unbonded) states for the Met80 recombination energetics. A full-dimensional surface-hopping algorithm based on the Landau-Zener theory is used to capture the nonadiabatic transitions between the bonded and unbonded states of the Met 80 ligand. The time evolution of Met 80 photodissociation and rebinding is explored through the simulation of multiple dissociation and rebinding trajectories. A distribution of rebinding time scales is observed and understood in terms of the dynamics of two key degrees of freedom, as well as the surrounding protein dynamics.

\section{METHODS}

PESs. Met80 rebinding involves at least two electronic states: ${ }^{1,4,11,20}$ the ground state singlet (s), associated with the bonded state, and the quintet (q), associated with the unbonded state. In this work, the classical CHARMM force field in the heme pocket has been extended by incorporating ab initio PES calculations. The force field has been designed to model these two spin states, including two sets of force parameters to describe the heme doming ${ }^{44,57-59}$ controlled by the $\mathrm{Fe}-\mathrm{N}$ bond, $\mathrm{N}-\mathrm{Fe}-\mathrm{N}$ angle, $\mathrm{S}-\mathrm{Fe}-\mathrm{N}$ angle, and $\mathrm{C}-\mathrm{S}-$ Fe angle interactions. ${ }^{11}$ The $\mathrm{Fe}-\mathrm{S}$ bond is modeled by a Morse potential

$$
V_{\mathrm{s}}(R)=D\left[\mathrm{e}^{-2 \alpha\left(R-R_{\mathrm{s}}\right)}-2 \mathrm{e}^{-\alpha\left(R-R_{\mathrm{s}}\right)}\right]
$$

for the singlet state and a repulsive potential

$$
V_{\mathrm{q}}(R)=A \mathrm{e}^{-2 \beta\left(R-R_{\mathrm{q}}\right)}-B
$$

for the quintet state, as functions of the $\mathrm{Fe}-\mathrm{S}$ distance R. $D$ is the $\mathrm{Fe}-\mathrm{S}$ binding energy, $R_{\mathrm{s}}$ is the equilibrium $\mathrm{Fe}-\mathrm{S}$ bond length, and $\alpha, \beta, A, B$, and $R_{\mathrm{q}}$ are parameters to be determined by fitting to $a b$ initio energy calculations and appealing to experimental observables.

PES Parametrization. Parameters for $V_{\mathrm{s}}$ and $V_{\mathrm{q}}$ were fitted using the following strategy. (1) A model system (Figure 2) of porphyrin, proximal imidazole ligand and distal dimethyl-sulfide ligand, modeling the Met80-heme-His18 complex, was employed in ab initio geometrical optimization to obtain a global minimum structure in a defined spin state. (2) Single point energy surface calculations, $V_{\text {Gaussian, }}$ were computed for both states by slowly changing $R$ with all other degrees of freedom fixed. (3) The same set of geometries obtained from (1) were optimized using the CHARMM force field to calculate Vcharmm, where Vcharmm is the total potential energy without the $\mathrm{Fe}-\mathrm{S}$ interaction. (4) $V_{\mathrm{s}}$ and $V_{\mathrm{q}}$ were fitted to the difference $V_{\text {Gaussian }}-V_{\text {charmm }}$. The idea of calculating single point energy is to minimize the effects from physically inaccurate deviations in secondary degrees of freedom due to the lower accuracy of the CHARMM force field when the system is far from the equilibrium configuration. Ab initio PES calculations were performed at the B3LYP/6-31G** level to the model system using Gaussian03. 


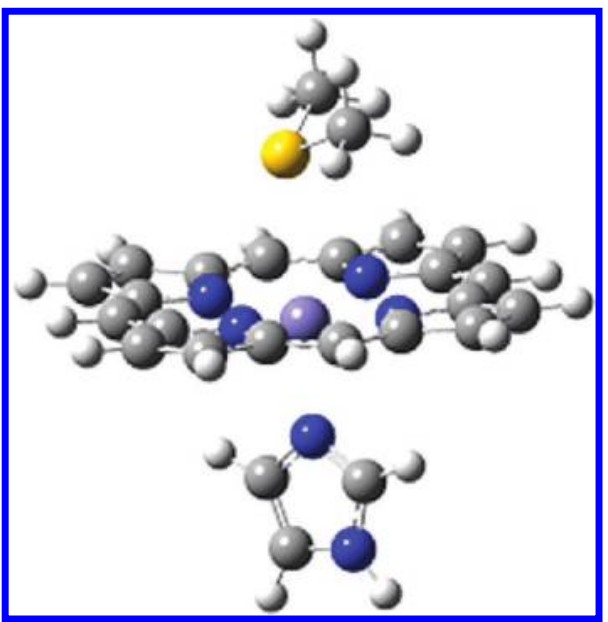

Figure 2. The model system for the truncated heme pocket including distal dimethyl-sulfide and proximal imidazole.

Adjustments to PES. The fundamental frequency of the $\mathrm{Fe}-$ $S$ stretching mode was found to be $372 \mathrm{~cm}^{-1} \cdot{ }^{60}$ Normal mode analysis was performed in CHARMM to capture and compare the $\mathrm{Fe}-\mathrm{S}$ stretching mode with experimental measurement. The parameters in eq 1 were adjusted accordingly to $D=14$ $\mathrm{kcal} / \mathrm{mol}, \alpha=2.6 \AA^{-1}$, and $R_{s}=2.32 \AA$.

In addition to the single point energy scan, an energy scan using full geometrical optimization of the model system at different $\mathrm{Fe}-\mathrm{S}$ distances was also performed. As shown in Figure 3, the dimethyl-sulfide group favors different orientations to the heme plane, leading to an up-and-down flipping motion, as it moves away from the heme. Calculations resulted in an $8 \mathrm{kcal} / \mathrm{mol}$ asymptotic energy difference between the singlet and quintet states, on the basis of which the parameter $B$ in eq 2 was adjusted. The final parameters in eq 2 were set to $A$ $=10 \mathrm{kcal} / \mathrm{mol}, B=3 \mathrm{kcal} / \mathrm{mol}, \beta=3.9 \AA^{-1}$, and $R_{\mathrm{q}}=2.3 \AA$.

Surface Hopping Algorithm. Simulation of chemical bond breaking and reformation requires the modeling of nonadiabatic transitions between different electronic states. The Landau-Zener ${ }^{46,47}$ theory of nonadiabatic surface-scattering is one theoretical model that has been applied to model ligand-
Fe binding in heme proteins. ${ }^{48}$ Zener's work in 1932 led to a result for the transition probability between two diabatic energy surfaces:

$$
P=\exp \left(-\frac{\pi^{2} \Delta^{2}}{h\left|\frac{\mathrm{d}}{\mathrm{d} t}\left(\varepsilon_{1}-\varepsilon_{2}\right)\right|}\right)
$$

where $\varepsilon_{1}$ and $\varepsilon_{2}$ represent the potential energies of two diabatic states. $\Delta$ is the electronic coupling between these two states assigned to the spin-orbit coupling in this case..$^{50,52,53} h$ is Plank's constant. This result is more commonly written as

$$
P=\exp \left(-\frac{\pi^{2} \Delta^{2}}{h v\left|F_{1}-F_{2}\right|}\right)
$$

where the time derivative of the energy difference between the diabatic states $\varepsilon_{1}$ and $\varepsilon_{2}$ is replaced by

$$
\frac{\mathrm{d} \varepsilon}{\mathrm{d} t}=\frac{\mathrm{d} \varepsilon}{\mathrm{d} R} \frac{\mathrm{d} R}{\mathrm{~d} t}=F v
$$

where $v$ is the velocity along the reaction coordinate, and $F_{1}$ and $F_{2}$ are the slopes of two potentials at the crossing point. Equation 4 is widely employed, as $F$ and $v$ can be easily determined for a one-dimensional system.

For the case of a transition between the singlet and quintet states involved in Met80 ligand binding in cyt c, the nonadiabatic transition is best treated as a multidimensional problem. In order to successfully implement eq 4 , one must reduce the PES to one or very few dimensions. This leads to an inaccurate description of the dynamics, as some of the essential reaction coordinates may be eliminated in the process of reduction. Another problem is potential energy discontinuity when a transition occurs. To conserve the total energy of the system, the potential energy change is usually deposited into the kinetic energy by rescaling the velocities of atoms around the reaction center, a process that is convenient but not necessarily physically reasonable.

We introduce a full-dimensional treatment of the LandauZener theory by appealing to eq 3 , eliminating the need to reduce the system to one dimension, as long as the first-order

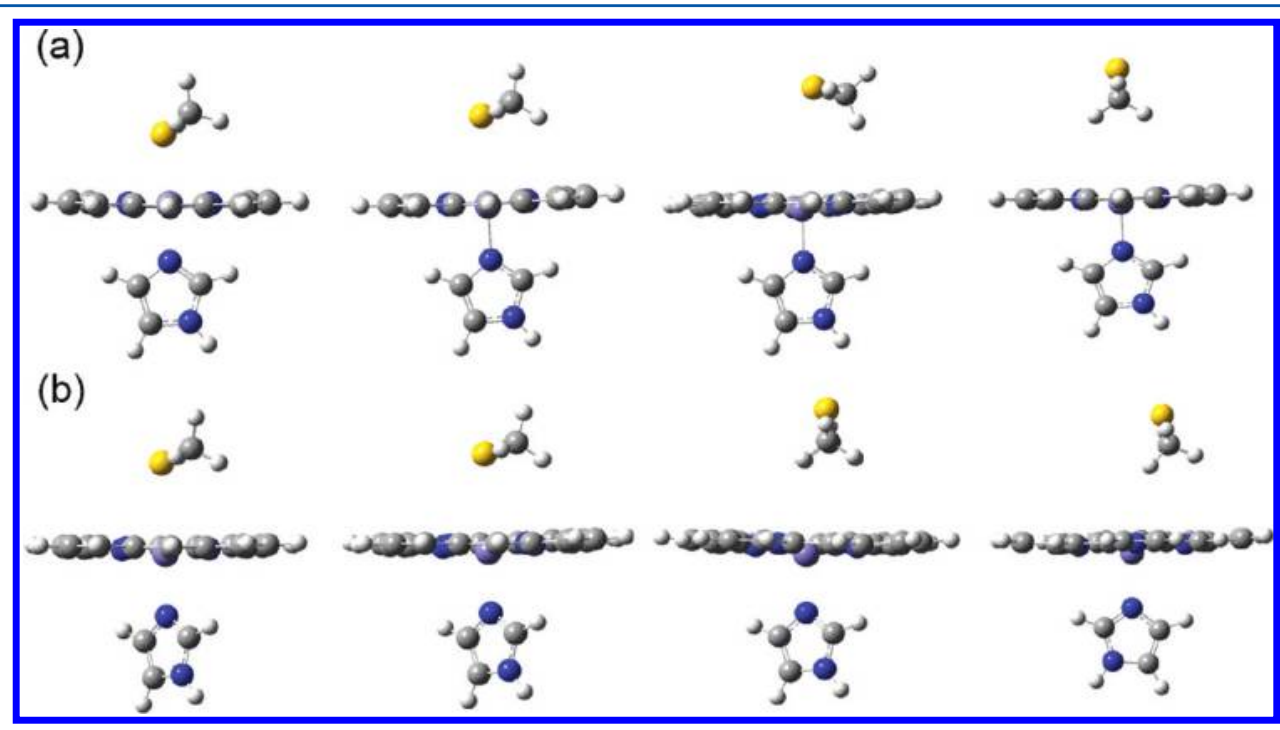

Figure 3. Geometrical optimizations of the model system at different $R_{\mathrm{Fe}-\mathrm{S}}$ : (a) singlet, from left to right, $R_{\mathrm{Fe}-\mathrm{S}}=2.44,3.2,4.4,5.2 \AA$; $(\mathrm{b})$ quintet, from left to right, $R_{\mathrm{Fe}-\mathrm{S}}=3.2,3.5,5.3,5.5 \AA$. 
derivative of the PES over time is known. We introduce an energy criterion to conserve the total energy of the system during transitions, simply based on the "physics" of a two-spinstate system. Spin flipping most likely occurs when the two states are close enough in energy, leading to large transition probability induced by strong spin-orbit coupling and a small energy barrier.

We define $\varepsilon_{\text {diff }}=\left|\varepsilon_{1}-\varepsilon_{2}\right|$ as the energy difference between the singlet state, $\varepsilon_{1}$, and the quintet state, $\varepsilon_{2}$. $\varepsilon_{1}$ and $\varepsilon_{2}$ are calculated at each time step by applying singlet and quintet force fields to the same geometry. When $\varepsilon_{\text {diff }}<\Delta \varepsilon$, a predefined small energy gap, the Landau-Zener transition probability $P$ is evaluated. $P$ is then compared to $r$, a random number from the uniform distribution between 0 and 1 . If $P>r$, a transition to the other spin state is accepted, otherwise, the system remains in the current state. In this work, the spin-orbit coupling, $\Delta$, is predetermined by appealing to calculations and experimental measurements in diatomic ligand-heme systems. ${ }^{50,61-63}$ The energy gap $\Delta \varepsilon$ influences the rebinding kinetics, and was with a value of no less than the maximum energy change between two consecutive time steps. $\Delta \varepsilon$ was defined to be $<2 \mathrm{kcal} / \mathrm{mol}$, and $\Delta$ was approximated as a constant set to be $350 \mathrm{~cm}^{-1}$. We have adjusted these parameters in a way that leads to kinetics for fast rebinding that are consistent with experimental observations, in order to probe the time scale and mechanism of the slower rebinding component.

MD Simulation. The sequence of $1 \mathrm{HRC}$ cyt $\mathrm{c}$ from the Protein Data Bank (PDB) was used to define the human cyt c system and the crystal structure was taken as the initial configuration. A cubic water box with 10440 TIP3P water molecules was added to surround the protein. The StormerVerlet algorithm and periodic boundary conditions were used, and the Ewald method was used to calculate the electrostatic energy. All bonds with hydrogen atoms were constrained using SHAKE. The simulation time step was 1 fs. The singlet state system (with 6-coordinated heme) was first minimized and then slowly heated up to $300 \mathrm{~K}$. A constant pressure and temperature (CPT) equilibration was performed for over $6 \mathrm{~ns}$. Seed structures were taken every 20 ps during the later stage of the CPT equilibration. The size of the water box was determined to be $68.8 \times 68.8 \times 68.8 \AA^{3}$. A total of 200 seed structures were collected and each was evolved for another 20 ps of constant volume and energy (NVE) equilibration. The final coordinates and velocities of each seed structure were collected as the initial coordinates and velocities for the dynamics simulations.

To simulate the photolysis process, the system underwent a vertical transition between the singlet (bonded) and the quintet (unbonded) force fields for each of the initial structures obtained from NVE equilibration. The system dynamics evolved at constant NVE. $\varepsilon_{\text {diff }}$ was calculated and monitored at each step. $P$ was calculated and compared with $r$ when the energy criterion for a transition was satisfied. Once a transition between electronic states was accepted, the force field was abruptly switched causing the system to undergo an energy change less than the magnitude of $\varepsilon_{\text {diff }}$ due to the occurrence of the transition. This energy change was ignored. Each initial structure was used to perform dynamics runs five times with a different random number, $r$, to compute the rebinding time statistics. A total of 1000 trajectories were collected for analysis.

\section{RESULTS}

Time Evolution of Photodissociation and Rebinding. The $\mathrm{Fe}-\mathrm{S}$ distance, $R_{\mathrm{Fe}-\mathrm{S}}$, was monitored as the key reaction coordinate and sensitive indicator of photodissociation and rebinding. Typical fast rebinding and slow rebinding trajectories are shown in Figure 4a. Each of the trajectories starts from a 6-

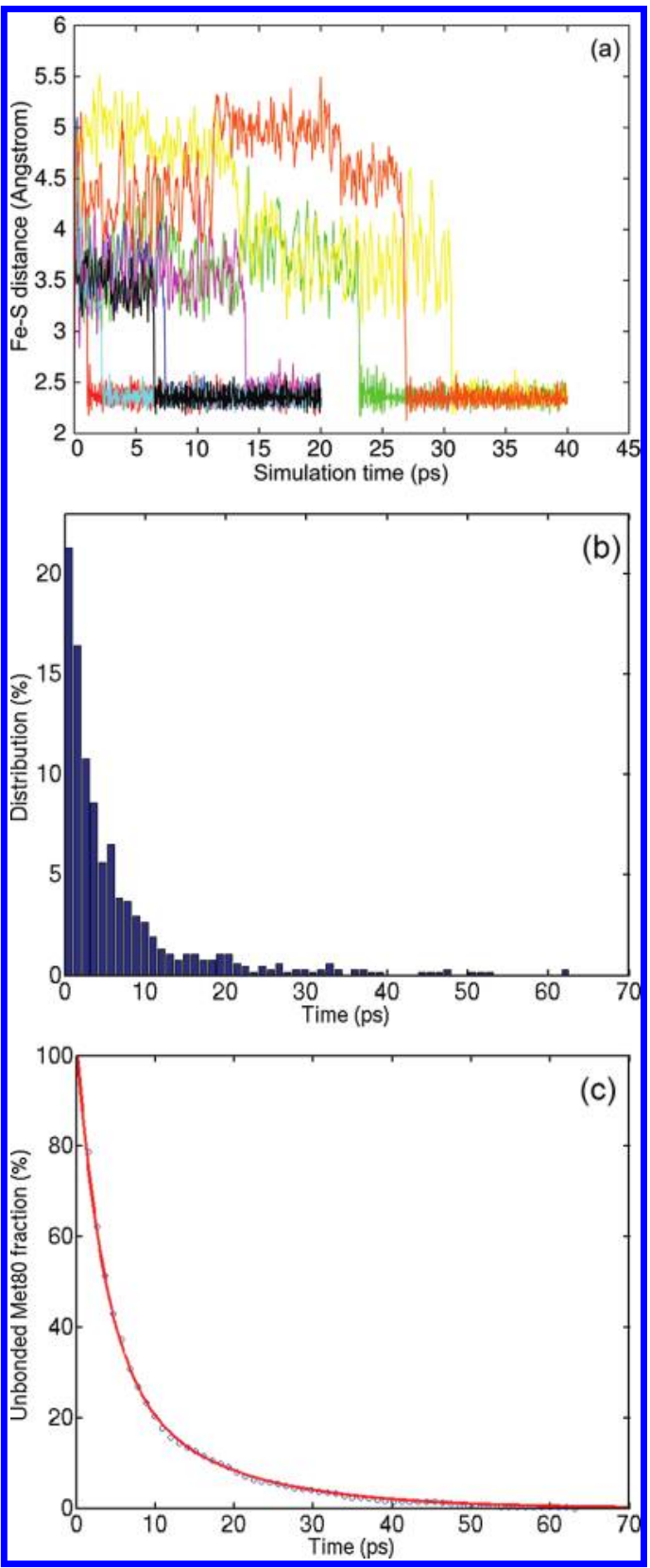

Figure 4. (a) Fast and slow rebinding trajectories, (b) histogram of rebinding times over 1000 trajectories, and (c) survival probability of unbounded Met 80 as a function of time.

coordinated equilibrium structure. For the fast rebinding trajectories, $R_{\mathrm{Fe}-\mathrm{S}}$ increases from the equilibrium bond length $2.3 \AA$ to $3.5-4 \AA$ within $100 \mathrm{fs}$ after photodissociation. Such a time is too short for nuclear reorganization, but sufficient for electronic density redistribution. In the transition, the Met80 ligand is immediately pushed away from the heme due to strong repulsive interactions. Upon rebinding, the $R_{\mathrm{Fe}-\mathrm{S}}$ then rapidly returns to $2.3 \AA$ after fluctuating around $3.5-4 \AA$ for the 
time scale of several picoseconds. The trajectories for the slow rebinding reveal the population and fluctuation of $R_{\mathrm{Fe}-\mathrm{S}}$ around $5 \AA$, and the $R_{\mathrm{Fe}-\mathrm{S}}$ is often observed to make a transition to intermediate distances of 3.5-4.5 Å before rebinding.

Figure $4 \mathrm{~b}$ shows the rebinding time distribution over 1000 trajectories. Figure $4 \mathrm{c}$ shows the fraction of unbounded Met80 over time, exhibiting a double exponential decay. Two major lifetime constants of unbounded Met80, $\tau_{1}, \tau_{2}$, were fitted using a linear combination of two single exponential functions to yield $u(t)=C_{1} \exp \left(-t / \tau_{1}\right)+C_{2} \exp \left(-t / \tau_{2}\right)$, where $C_{1}=70.4, C_{2}$ $=30.2, \tau_{1}=3.6 \mathrm{ps}$, and $\tau_{2}=14.4 \mathrm{ps}$. This defines two time scales: $\tau_{1}$ for fast rebinding and $\tau_{2}$ for slow rebinding.

Mechanism of Multistep Rebinding. A key goal of our study is to resolve the mechanism of rebinding associated with the different rebinding times observed in the doubleexponential decay. The dissociated Met80 ligand in cyt $c$ is covalently connected to the protein backbone making the reaction unimolecular in the case of which the protein dynamics are restricted to fluctuations among conformational substates of the native structural ensemble. In our simulations, multiple conformational substates of the dissociated Met 80 were found to be essential to the interpretation of the fast and slow rebinding time scales observed in experimental studies.

We have observed that the dissociated methionine samples specific conformational substates before it rebinds to the heme Fe. Figure $5 b, c$ shows two different substates with respect to the $\mathrm{C}-\mathrm{S}-\mathrm{C}$ pointing downward and upward. Figure 5a shows the orientation of the $\mathrm{C}-\mathrm{S}-\mathrm{C}$ when it is bonded to the Fe. Comparison of panels $a$ and $b$ reveals that the downward substate is conformationally similar to the bonded state, except that the $\mathrm{Fe}-\mathrm{S}$ distance is larger and the sulfur atom is slightly tilted up. Comparing panels $b$ and $c$, one can see that the upward substate differs from the bonded state through a reorientation of the $\mathrm{C}-\mathrm{S}-\mathrm{C}$ group. The $\mathrm{Fe}-\mathrm{S}$ distance reaches its longest separation at this point. Detailed analysis on 1000 trajectories suggests that the population of these conformational substates leads to different rebinding time scales.

Another major conformational difference between the dissociated states and the bonded state is the heme doming with the Fe out-of-plane displacement. This can be observed from Figure 5b,c, with a noticeable out-of-plane displacement of the heme $\mathrm{Fe}$, as opposed to the in-plane $\mathrm{Fe}$ atom observed in Figure 5a.

The reaction system can be represented in terms of a twodimensional reaction coordinate distinctly separated from the coupled bath of surrounding protein and solvent degrees of freedom. It is difficult to describe the orientation of the active site, including relative orientation of Met80 to the heme, exclusively through the internal coordinates of Met80. One approach would be to consider combinations of torsional angles $\mathrm{C}-\mathrm{S}-\mathrm{C}-\mathrm{C}, \mathrm{S}-\mathrm{C}-\mathrm{C}-\mathrm{C}$, or $\mathrm{C}-\mathrm{C}-\mathrm{C}-\mathrm{C}$ of the Met80 that reflect the rotamers of the Met80. Those combinations of torsional angles considered as generalized coordinates led to a wide variety of values that were not well correlated with the upward and downward states that are associated with the reaction intermediates. Two methionine rotamers studied by Yamashita et al. ${ }^{7}$ have also been observed in our MD simulations. In our study, those two rotamers were found in both the bonded and dissociated states, and have no direct correlation with the $\mathrm{C}-\mathrm{S}-\mathrm{C}$ downward and upward orientations in the dissociated state in particular. One example is Figure $5 a-c$, which illustrates one type of methionine rotamer that exists in the bonded state, the dissociated

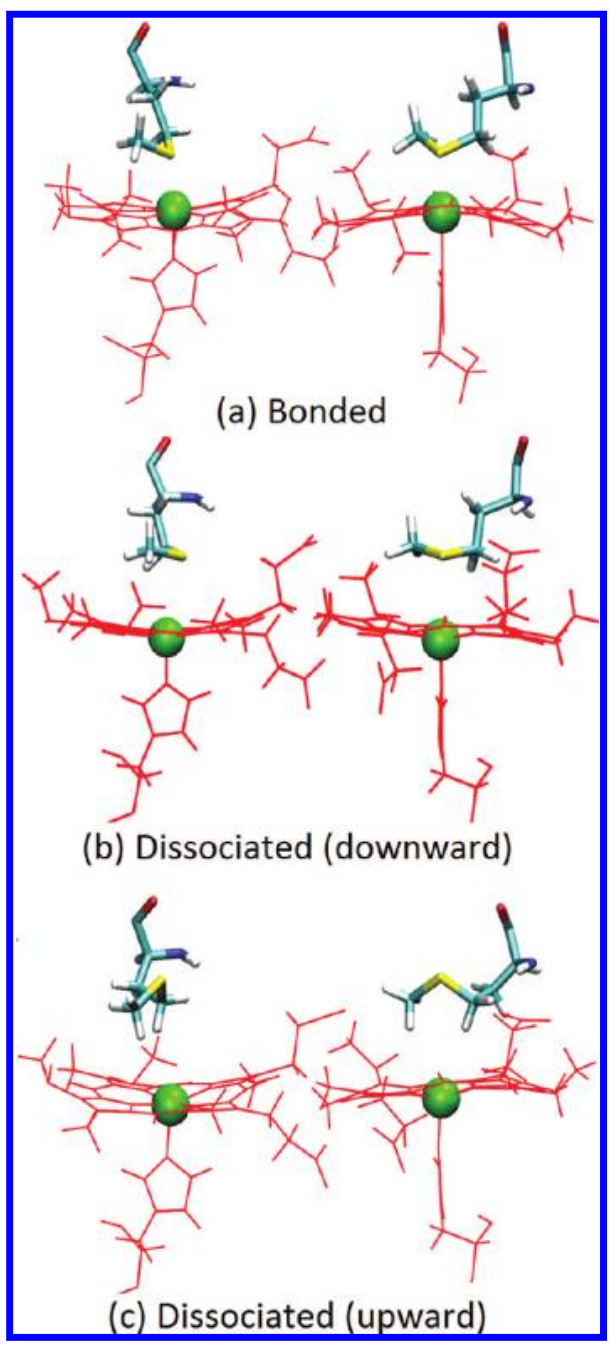

Figure 5. (a) Bonded Met 80 configuration and dissociated Met 80 configurations (head on and rotated 90 degrees) in (b) the downward substate and $(c)$ the upward substate.

downward state, and the dissociated upward state. In order to show the correspondence between the conformational substates and rebinding times, the downward and upward orientations in Figure 5 are alternatively represented as an angle $\theta$ describing the $\mathrm{C}-\mathrm{S}-\mathrm{C}$ flipping motion of the Met80 and the Fe-S distance $R_{\mathrm{Fe}-\mathrm{S}}$. As shown in Figure 6, $\theta$ is defined as the angle between two vectors, $r_{1}$ and $r_{2} . r_{1}$ is defined by the iron atom and the nitrogen atom of the histidine residue that is connected to the iron atom. $r_{2}$ bisects the $\mathrm{C}-\mathrm{S}-\mathrm{C}$ angle of the Met80. The $R-\theta$ two-dimensional trajectories of six fast and two slow rebinding events are shown in Figure 7. For the fast rebinding trajectories, $\theta$ populates two main regions (where $R_{\mathrm{Fe}-\mathrm{S}}=3.5-4 \AA$ ) with a slight change of roughly $20^{\circ}$ compared to the $\theta$ values in the equilibrium region (where $R_{\mathrm{Fe}-\mathrm{S}}=2.3 \AA$ ). For the slow rebinding trajectories, $\theta$ populates three different regions. In one region, $R_{\mathrm{Fe}-\mathrm{S}}$ is large $(5 \AA)$ with a roughly $100^{\circ}$ rotation of $\theta$ from the equilibrium position, indicating a flipping from downward to upward. The other two regions are merged into an area where $R_{\mathrm{Fe}-\mathrm{S}}$ is around 3.5-4 $\AA$. The time needed for transition between the upward and downward orientations of $\theta$ is the main cause of a slower rebinding.

In Figure 8, the overlap of a fast and a slow rebinding twodimensional trajectory shows the differences in terms of the changes of $\theta$ and $R_{\mathrm{Fe}-\mathrm{S}}$. In the fast rebinding trajectory, the 


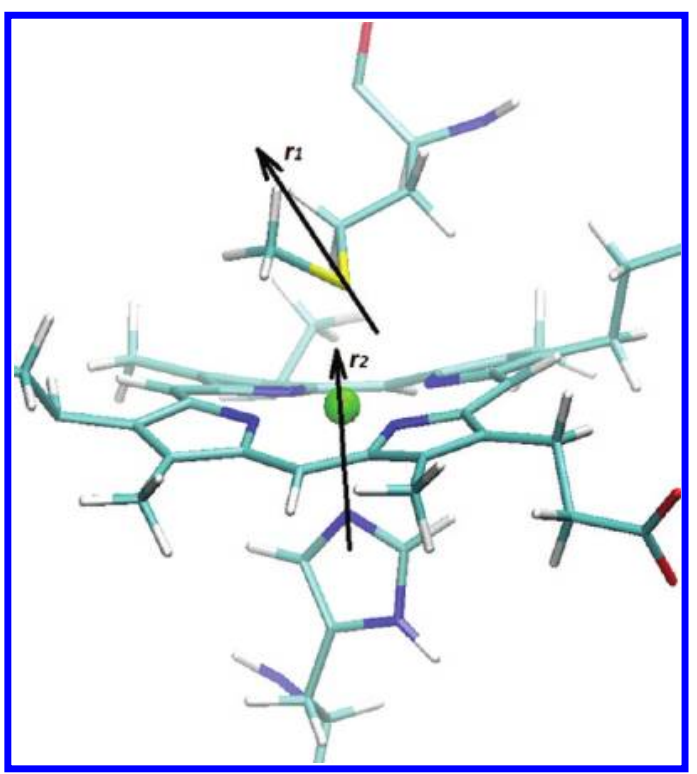

Figure 6. Definition of reaction coordinate $\theta$ as the angle between vectors $r_{1}$ and $r_{2}$.

motion of the Met80 is relatively restricted to the downward substate. For the slow rebinding trajectory, the Met 80 is able to move more freely to populate the downward and upward substates. The three dimensional schemes of trajectories in Figure 7 are shown in Figure 9 in terms of the time evolution of two key reaction coordinates $\theta$ and $R_{\mathrm{Fe}-\mathrm{S}}$. Adding the time axis (vertical axis) to reaction coordinates $R$ and $\theta$ (horizontal axes) provides insight into how the system transitions from one substate to another. Each trajectory starts from the bottom with dark blue color, and, as time evolves, the color changes become warmer and warmer. The projection of the trajectories on the $R_{\mathrm{Fe}-\mathrm{S}}-\theta$ plane is identical to the corresponding plot in Figure 7 .

To explore the nature of configurations in the unbonded state, a 3.2 ns equilibration of the 5-coordinated system (with dissociated Met80) in the quintet state was performed and the distributions of $R_{\mathrm{Fe}-\mathrm{S}}$ as well as $\theta$ are shown in Figure 10a,b. In each plot of Figure 10, three Gaussian functions were used in the fit with the sum of the Gaussians shown in red. The center of each function is set to $R_{\mathrm{Fe}-\mathrm{S}}=3.5 \AA, 4.0 \AA$, and $5 \AA$, and $\theta=$ $50^{\circ}, 75^{\circ}$, and $170^{\circ}$, respectively, defining three distinct conformational substates. The dissociated system differentially samples those substates, resulting in different rebinding time scales when it comes to a reactive dynamics run. Figure 10 provides insight into the population of each substate, where the fast rebinding contribution from downward substates comprises over $90 \%$ of the population, and the remaining population is assigned to the upward substate.

Free Energy Landscape and Kinetic Energy Relaxation. Although two key reaction coordinates have been extracted to study the rebinding dynamics, there are many other degrees of freedom involved that are, in general, difficult to explicitly describe. Rather than using the multidimensional potential energy landscape of the protein, a lower dimensional effective free energy landscape may be defined to provide insight into the kinetics of the system at finite temperature. By binning data from trajectories (as in Figure 7), a probability density distribution $P(R, \theta)$ may be defined along with the effective free energy ${ }^{9}$

$$
F(R, \theta)=-k_{\mathrm{B}} T \ln P(R, \theta)
$$
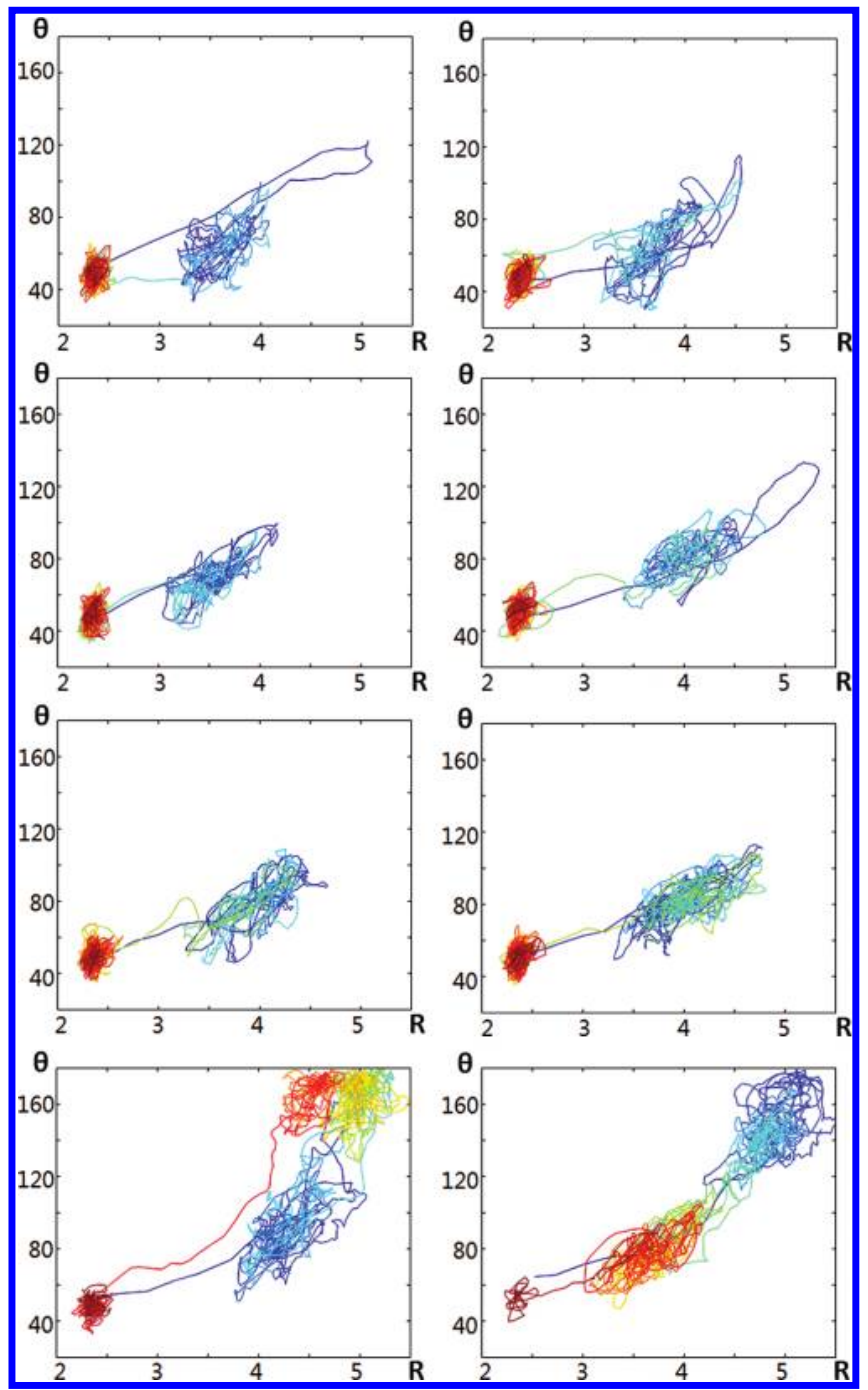

Figure 7. Two-dimensional projections of trajectories of fast and slow rebinding of Met80 ligand ( $\theta$ in degrees, $R$ in angstroms). The trajectory is initially dark blue, and evolves as time progresses to be light blue, green, yellow, orange, and finally red.

where $k_{\mathrm{B}}$ is the Boltzmann's constant and $T$ is temperature. Figure 11a shows the effective free energy surface of the reactive system as a function of two reaction coordinates, $R$ and $\theta$, as well as the two-dimensional contour projection on the $R-\theta$ plane. From this free energy landscape, the minimum energy path was computed with the result shown in Figure $11 \mathrm{~b}$. The reaction path shows two reactant states (corresponding to the downward and upward conformational substates) and one product state (corresponding to the bonded state). The energy barrier for the transition from the upward substate to the downward substate is $0.5 \mathrm{kcal} / \mathrm{mol}$ in the forward direction and $2 \mathrm{kcal} / \mathrm{mol}$ in the backward direction. The barrier for the transition from the downward substate to the product state is 1 $\mathrm{kcal} / \mathrm{mol}$. The barrier from the product state to the downward substate is $>3 \mathrm{kcal} / \mathrm{mol}$ and $>4 \mathrm{kcal} / \mathrm{mol}$ to the upward substate. There is a natural flow of probability from the upward to downward substates. Figure 7 provides an example of transition from the downward substate to the upward substate, which is possible but rare. It is highly unlikely to have a transition from the product state to the reactant states due to a large energy gap. Recrossing of the barrier from the product state to the reactant states after rebinding is never observed in 


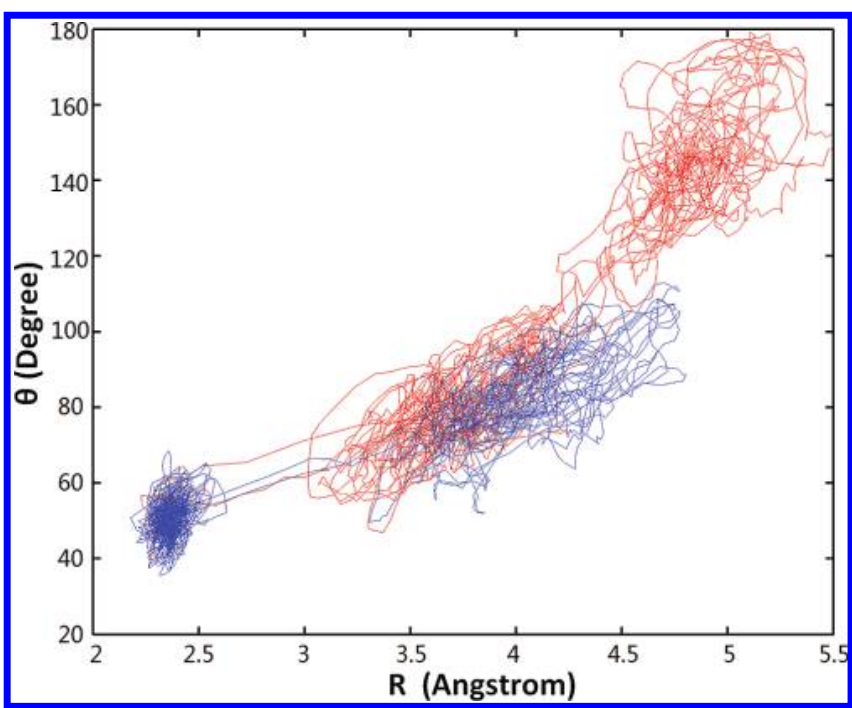

Figure 8. An overlap of two-dimensional projections of fast (blue) and slow (red) rebinding trajectories for Met 80 ligand. Relatively restricted motion of the Met80 ligand and lack of conformational transition in angle $\theta$ is observed to underlie fast rebinding.

the MD simulations. In fact, thermal dissociation of axial ligands is known to take place in the microsecond time scale. Therefore, dissociation was not expected to be observed in our picosecond time scale simulations. Such a large energy gap can only be overcome by developing a high concentration of excess energy through, for example, photodissociation that breaks the valence bond and causes a vertical transition.

In order to examine the feasibility of applying some form of equilibrium reaction rate theory, it is useful to look at the time scales of kinetic energy relaxation along those key reaction coordinates following the photodissociation. Figure 12a,b shows the 20 ps kinetic energy relaxations of the heme and Met 80 as well as the time autocorrelation function. $E$ is defined as the total kinetic energy of all heavy atoms in each residue and $\langle E\rangle=3 N k_{\mathrm{B}} T / 2$ as the average equilibrium kinetic energy, where $N$ is the number of heavy atoms in each residue. The kinetic energy relaxation of the Met80 occurs in less than 1 ps, and is substantially slower (on the order of several picoseconds) for the heme. The heme pocket is able to reach equilibrium or near equilibrium when the rebinding occurs (with a 3.6 ps time scale for fast rebinding and 14.4 ps for slow rebinding), suggesting that it may be possible to apply equilibrium reaction rate theory to estimate the reaction rate.

Fe Out-of-Plane Motion. Experimentalists have observed that the $\mathrm{Fe}$ atom moves out of the heme plane following photodissociation. ${ }^{4,35,59,64}$ This has also been observed in our MD simulations as well as ab initio geometrical optimizations. Figure $3 \mathrm{~b}$ shows several optimized structures in the quintet state at different $\mathrm{Fe}-\mathrm{S}$ distances. The $\mathrm{Fe}$ atom moves out of plane within a very short time, with the separation of the dimethyl-sulfide group and the heme occurring more gradually. This indicates that the Fe out-of-plane motion is induced by the instantaneous change of the local electronic structure associated with photodissociation. For the case of the singlet state, in Figure $3 \mathrm{a}$ the $\mathrm{Fe}$ atom stays in plane except for a slight displacement at large $\mathrm{Fe}-\mathrm{S}$ distance. A large $\mathrm{Fe}-\mathrm{S}$ separation at low spin state (singlet) is a physically unfavorable high-energy configuration upon photodissociation. Figure 13 shows the distribution of $\mathrm{Fe}-\mathrm{S}$ distances in the singlet and quintet states from the rebinding dynamics simulations. $R_{\mathrm{Fe}-\mathrm{S}}$ is strongly localized around $2.3 \AA$ in the singlet state with an upper bound no greater than $3.2 \AA$. This suggests that the slight out-of-plane displacement of the $\mathrm{Fe}$ atom at large $R_{\mathrm{Fe}-\mathrm{S}}$ may be ignored for the singlet state force field. $R_{\mathrm{Fe}-\mathrm{S}}$ reveals a wider distribution in the quintet state, as the dissociated Met 80 samples a number of conformational substates, including torsional transitions in the Met80 side chain.

\section{DISCUSSION}

Met80 Side Chain Transitions. The flipping motion of the Met80 has been observed in MD simulations of Met80 dissociation and rebinding (see Figure 5) and interpreted as the essential mechanism underlying the fast and slow rebinding dynamics. In the model system of porphyrin-dimethyl sulfide, the orientational transition in the dimethyl sulfide group with increasing $\mathrm{Fe}-\mathrm{S}$ distance observed in Figure 3 provides insight into the rebinding mechanism. The methyl groups and heme maintain a strong interaction despite the spin state change. During dissociation of dimethyl sulfide (Figure 3), the methyl groups are pulled toward the heme and remain at a relatively static distance (about $3.75 \AA$ ) from the heme (see Table 1). The attraction between the sulfur atom and the heme decreases as $R_{\mathrm{Fe}-\mathrm{S}}$ increases in the singlet state, and turns into a strong repulsion when the spin switches to quintet upon dissociation. The sulfur atom therefore gains substantial momentum to create a significant separation from the heme. Similar attraction interactions between the terminal methyl group of Met80 and the heme, as well as the s-flanking methylene of Met80 and the heme, also appear to stabilize the upward dissociated configuration.

Surface Hopping Dynamics and Assumptions. The energy criterion in this Landau-Zener-based surface hopping algorithm ensures that the spin state change is only allowed when the potential energies of each state, $\varepsilon_{1}$ and $\varepsilon_{2}$, are sufficiently close. The total energy of the system is conserved without a need for redistribution of energy over the system modes to address unphysical discontinuities in the potential energy. Figure 14 shows three trajectories in which $\varepsilon_{1}, \varepsilon_{2}$, and the actual potential energy of the system at each time step, $\varepsilon_{\text {actual, }}$ are monitored. One can see that $\varepsilon_{\text {actual }}$ smoothly transitions between $\varepsilon_{1}$ and $\varepsilon_{2}$. The allowed energy jump is approximately the magnitude of the electronic coupling between the two states. Figure 14 also shows multiple hopping transitions between the two electronic states. The approximate surface hopping method employed in this study aims to provide a dynamics that captures the overall statistics of surface crossing. In that sense, the detailed dynamics of multiple surface hopping in a given encounter in the transition region may correspond to a more complex quantum dynamics where there is a significant probability of population on each diabatic surface as the nuclei pass through the crossing region and before they separate into the better defined asymptotic states. The heme doming motion, as well as the distance and relative orientation between the Met80 and heme, are the major conformational "order parameters" that define the transition region. The sulfur atom in Met80 must approach the $\mathrm{Fe}$ atom with the Met80 residue in a downward conformation of rebinding to occur. The heme doming configuration at the time of rebinding tends to be flat as opposed to being domed toward the His18. Many other conformational factors could be taken into account in order to provide a more fine-grained description of the system conformation. However, the two 

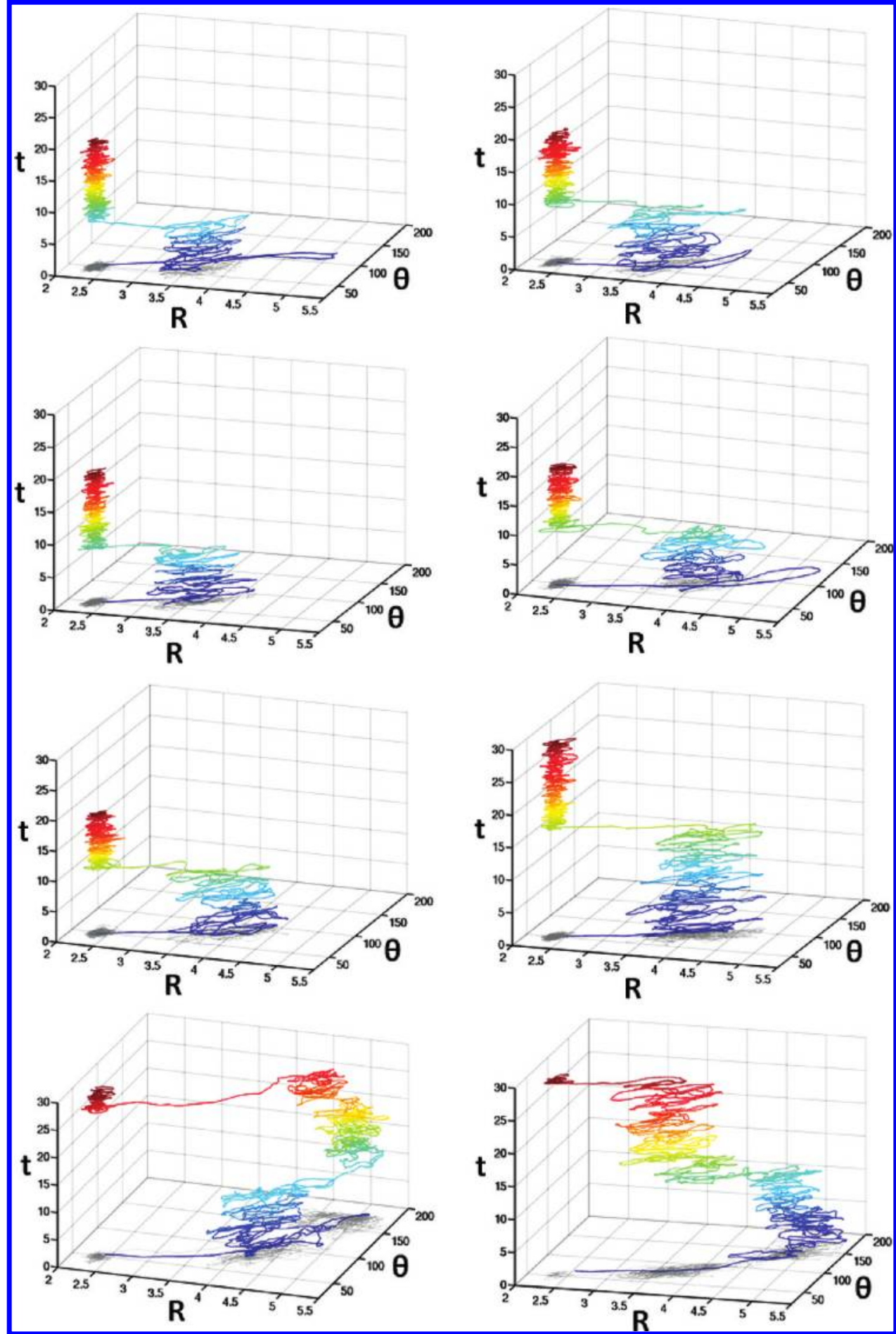

Figure 9. Time evolution of Met80 rebinding trajectories displayed in three dimensions of $R, \theta$, and, on the vertical axis, time ( $R$ in angstroms, $\theta$ in degrees, and time in ps).

principal coordinates that we have defined capture what we feel are the essential degrees of freedom that describe the slow and fast rebinding dynamics.

Although our model appears to capture essential aspects of the methionine rebinding mechanism, it is an approximate and simplified model of the nonadiabatic reaction dynamics. The time dependence of the PES in the crossing zone is a linear approximation. This assumption excludes the case in which two PESs approach but never cross. In that case, the transition probability can be significantly different from the linear model.
One of the theories to describe this noncrossing case is the Rosen-Zener model. ${ }^{65,66}$ An alternative model is the Demkov model. ${ }^{67}$ In those models, the electronic coupling is treated exponentially as a function of either the time or the reaction coordinate. $^{65-68}$

Electronic coupling of a system that involves spin state change is reduced to the spin-orbit coupling, which is a challenge to address both theoretically and experimentally, even in cases where the initial and final electronic states and energy surfaces are well-defined. The actual coupling is time-depend- 


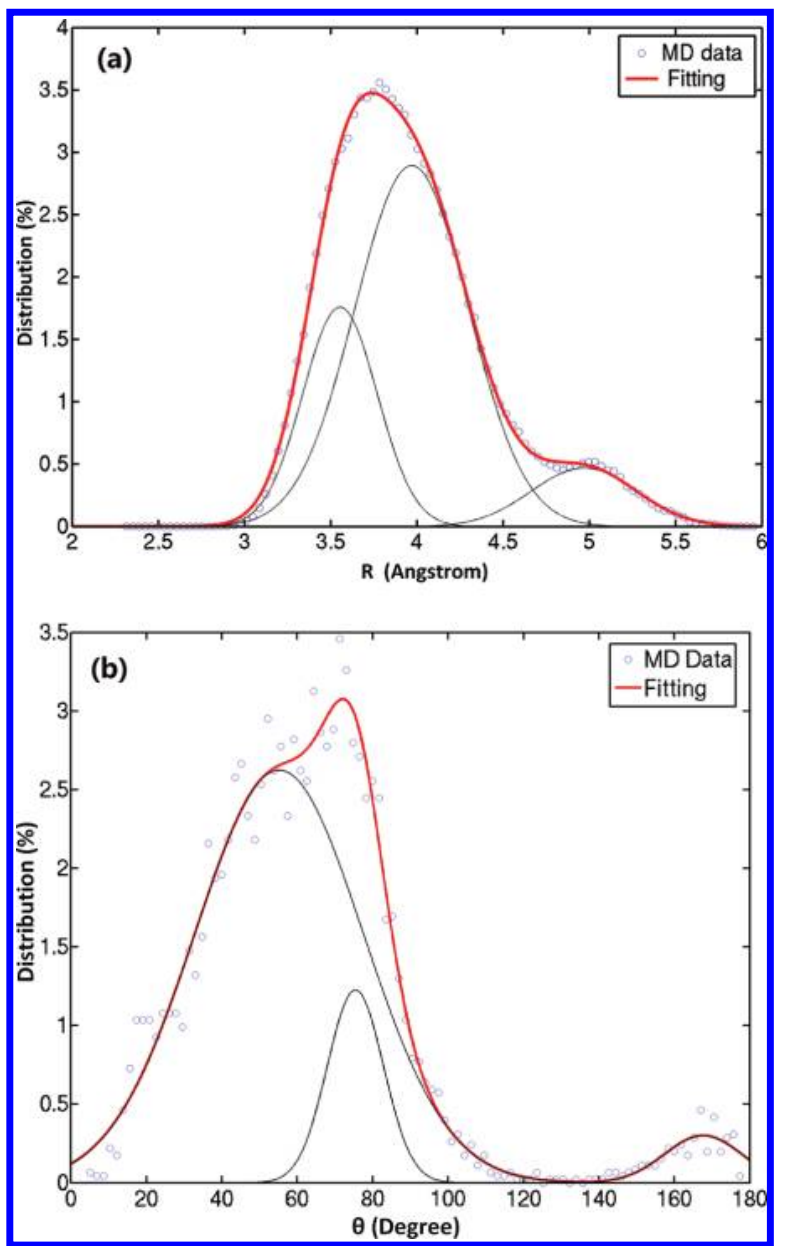

Figure 10. (a) $R_{\mathrm{Fe}-\mathrm{S}}$ and (b) $\theta$ probability distributions from sampling conformations of the protein in unbonded quintet state only (no transitions to the singlet state for rebinding were allowed). The simulations in the absence of dissociation or rebinding clearly show three major conformational substates in each of the distributions.

ent (conformation-dependent) and cannot be easily calculated during MD simulations. The coupling is treated as a constant in time in the Landau-Zener linear model, as the crossing zone of the PES is sufficiently small. Our energy criterion restricts the coupling to being estimated only within the crossing zone.

Direct transitions between singlet and quintet states are spinforbidden. ${ }^{39,40,61}$ However, transitions can still be induced by a strong spin-orbit coupling. ${ }^{54}$ Sometimes the multiple-step rebinding process can be caused by multiple-step spin state change, for example, a quintet-triplet-singlet process. An ab initio study of a spin-dependent mechanism for diatomic ligand binding to heme by Franzen ${ }^{61}$ provides a thorough discussion of this point. In our work, this multiple-step spin flipping was not considered as a dominant mechanism, as the electronic structural relaxation happens in such a short time that any intermediate spin state would be too short-lived to be considered populated in our dynamics.

Limitations of Force Field Parametrization. We have presented an extended CHARMM force field in the heme pocket that leads to efficient and reasonable dynamics. However, a full set of force field parameters for both spin states must be designed to provide a better description of the nonadiabatic rebinding dynamics as well as to elucidate the intramolecular vibrational energy relaxation in heme proteins.
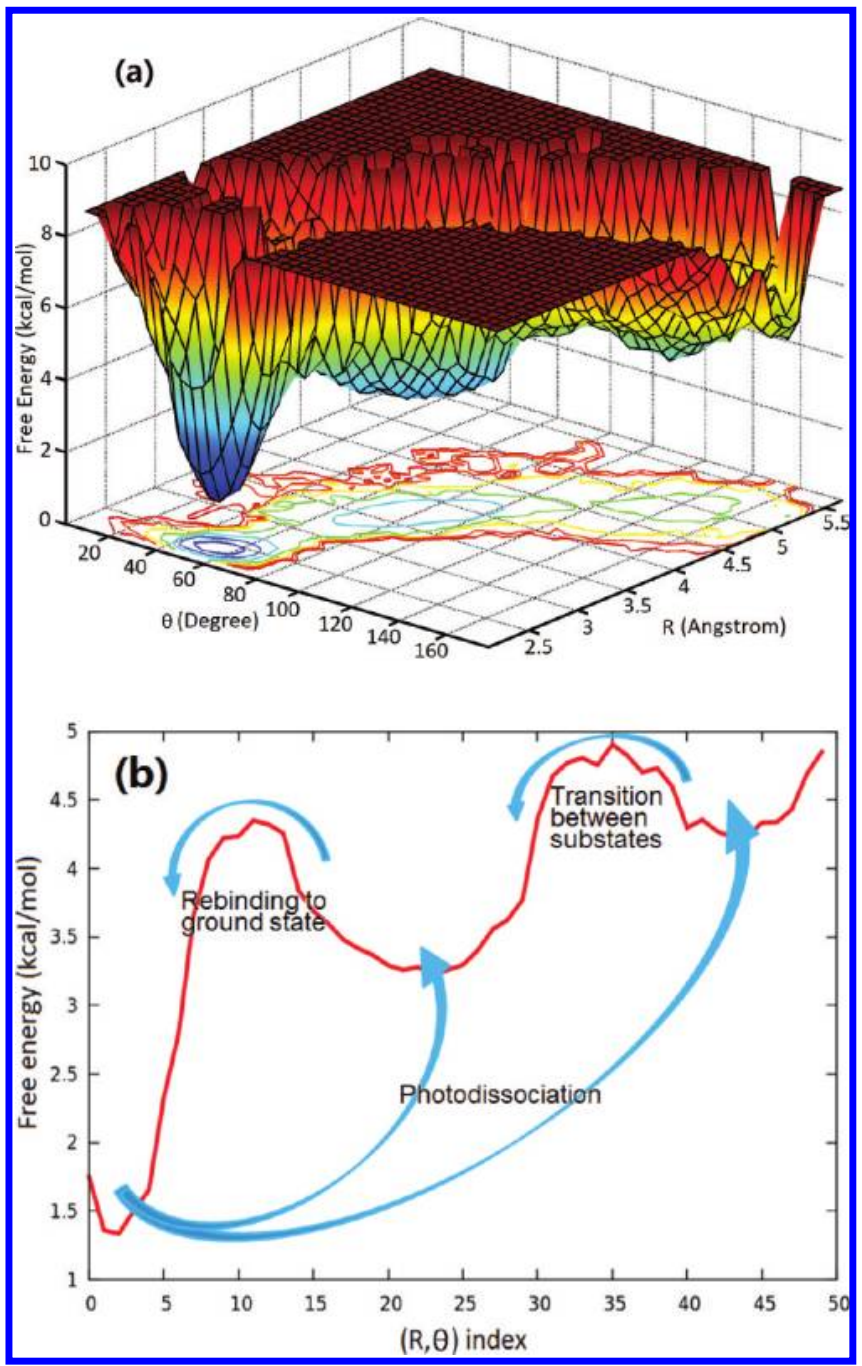

Figure 11. (a) Effective free energy landscape computed from observed rebinding trajectories and (b) associated one-dimensional minimum energy pathway.

In this work, the force field parametrization for the bonded and dissociated states has been strictly limited in terms of heme doming and $\mathrm{Fe}-\mathrm{S}$ interaction. All other terms were treated as in the CHARMM force field. This includes the partial charges on each atom in the heme pocket as well as some angle and torsion terms ${ }^{69}$ that have been tested in our study. The same set of partial charges were employed for both singlet and quintet states in our study. Luthey-Schulten ${ }^{70}$ and co-workers have presented an alternative set of partial charges of the singlet Met80-heme-histidine (His18) pocket, including changes in bond, angle, and torsion parameters. Meuwly and co-workers have carried out similar work for the quintet state (personal communication from Meuwly). A rigorous self-consistent parametrization of both the singlet and quintet states for this system is a goal for future studies.

\section{CONCLUSION}

An ab initio incorporated CHARMM force field has been extended to the heme pocket in cyt $\mathrm{c}$ to study the dynamics of Met80 ligand rebinding. A surface hopping algorithm based on Landau-Zener theory has been applied to address the nonadiabatic transition dynamics. A two-step rebinding mechanism has been proposed and described in terms of the 


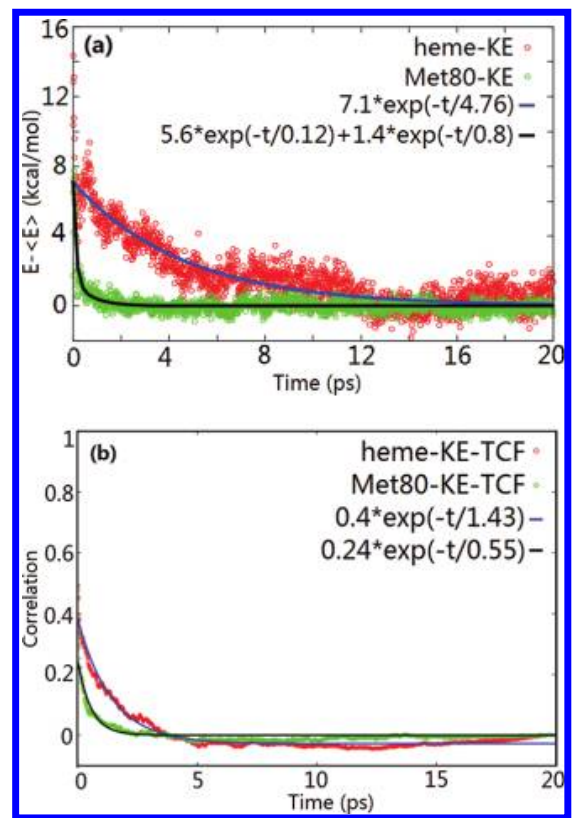

Figure 12. (a) Kinetic energy relaxation of heme and Met80 in the first $20 \mathrm{ps}$ following ligand photodissociation and (b) time autocorrelation functions of the kinetic energies of heme and Met80 in the first 20 ps.

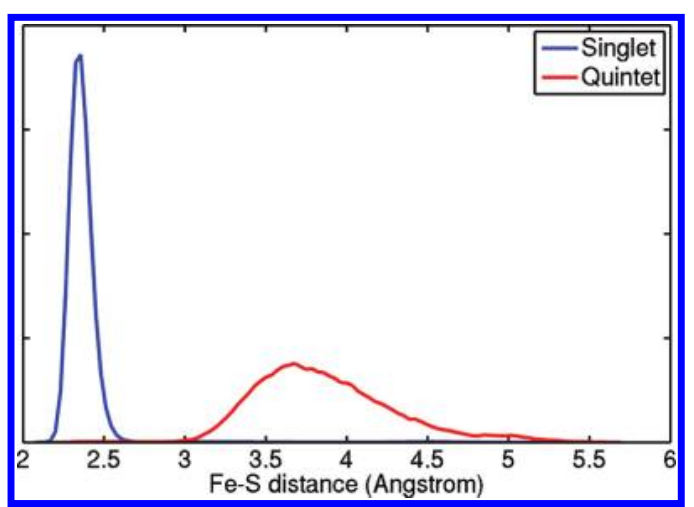

Figure 13. $R_{\mathrm{Fe}-\mathrm{S}}$ distributions in singlet and quintet states derived from rebinding dynamics trajectories.

Table 1. Key Structural Parameters of the Dimethyl SulfidePorphyrin-Imidazole Model System

\begin{tabular}{|c|c|c|}
\hline $\mathrm{Fe}-\mathrm{S}$ distance $(\AA)$ & Fe oop ${ }^{a}$ displacement $(\AA)$ & C-heme distance ${ }^{b}(\AA)$ \\
\hline 3.2 & 0.18 & 3.65 \\
\hline 3.5 & 0.22 & 3.75 \\
\hline 5.3 & 0.324 & 3.77 \\
\hline 5.5 & 0.327 & 3.76 \\
\hline
\end{tabular}

${ }^{a}$ Out-of-plane. ${ }^{b}$ Average distance between methyl carbons and heme plane.

population of three major conformational substates of the dissociated Met80 ligand. The $\mathrm{Fe}$ out-of-plane displacement and the $\mathrm{C}-\mathrm{S}-\mathrm{C}$ group flipping of the Met80 are found to be essential functional motions of the system that are essential to ligand dissociation and recombination dynamics. The overall dynamics is consistent with prior experimental studies and provides an atomic-level interpretation of the mechanism underlying the distribution of time scales and the connection between the protein structure and function.
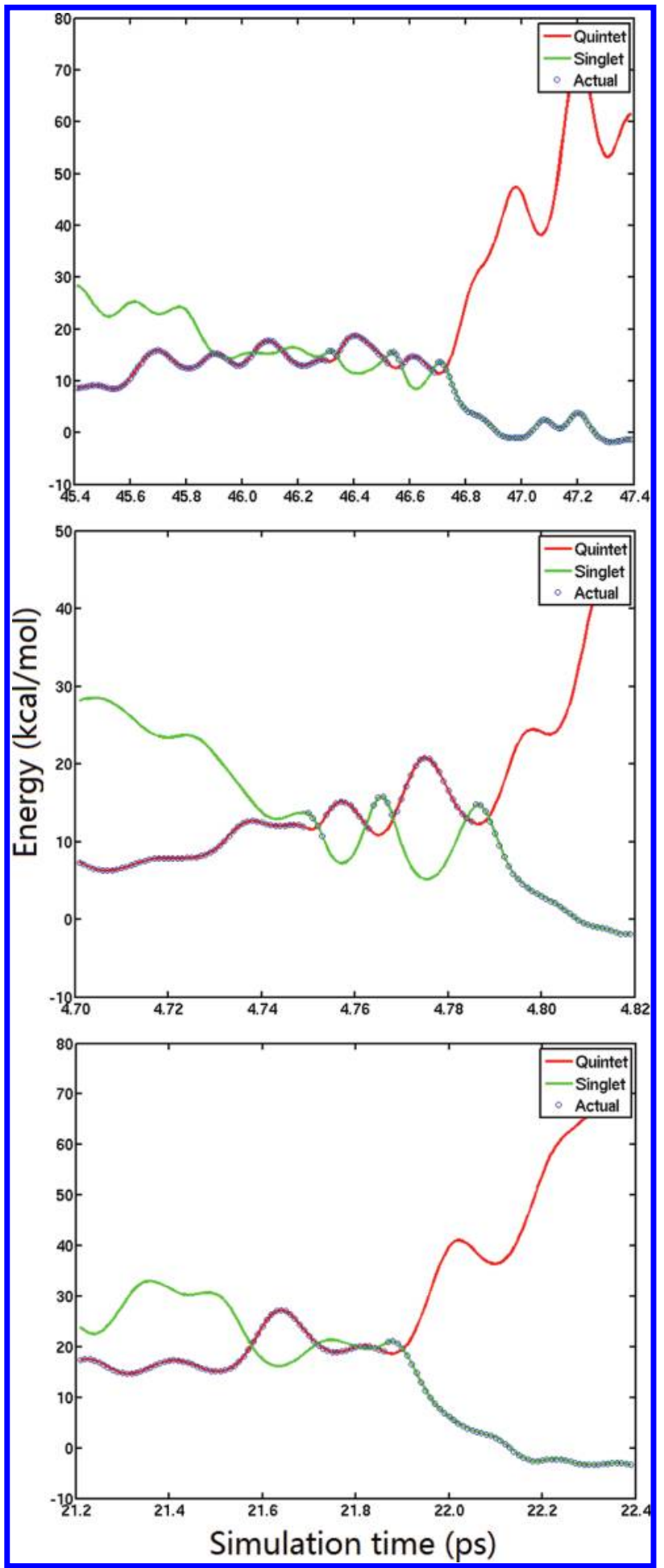

Figure 14. Observed transitions between electronic states projected on the time-dependent PES during Met80 rebinding dynamics for three trajectories.

\section{AUTHOR INFORMATION}

\section{Corresponding Author}

*E-mail: straub@bu.edu.

\section{Notes}

The authors declare no competing financial interest. 


\section{ACKNOWLEDGMENTS}

We are grateful for the generous support of our research by the National Science Foundation (CHE-114676 and CHE0750309) and the Boston University Center for Computational Science.

\section{REFERENCES}

(1) Negrerie, M.; Kruglik, S. G.; Lambry, J. C.; Vos, M. H.; Martin, J. L.; Franzen, S. J. Biol. Chem. 2006, 281, 10389-10398.

(2) Walther, M.; Raicu, V.; Ogilvie, J. P.; Phillips, R.; Kluger, R.; Miller, R. J. J. Phys. Chem. B 2005, 109, 20605-20611.

(3) Silkstone, G.; Jasaitis, A.; Wilson, M. T.; Vos, M. H. J. Biol. Chem. 2007, 282, 1638-1649.

(4) Mizutani, Y.; Kitagawa, T. Chem. Rec. 2001, 1, 258-275.

(5) McMahon, B. H.; Stojkovic, B. P.; Hay, P. J.; Martin, R. L.; Garcia, A. E. J. Chem. Phys. 2000, 113, 6831-6850.

(6) Margulis, C. J.; Guallar, V.; Sim, E.; Friesner, R. A.; Berne, B. J. J. Phys. Chem. B 2002, 106, 8038-8046.

(7) Yamashita, T.; Bouzhir-Sima, L.; Lambry, J. C.; Liebl, U.; Vos, M.

H. J. Biol. Chem. 2008, 283, 2344.

(8) Agmon, N.; Hopfield, J. J. J. Chem. Phys. 1983, 79, 2042.

(9) Nutt, D. R.; Karplus, M.; Meuwly, M. J. Phys. Chem. B 2005, 109, 21118-21125.

(10) Berlina, Y. A.; Fischer, S. F.; Chekunaevb, N. I.; Goldanskii, V. I. Chem. Phys. 1995, 200, 369-385.

(11) Meuwly, M.; Becker, O. M.; Stote, R.; Karplus, M. Biophys. Chem. 2002, 98, 183-207.

(12) Banushkina, P.; Meuwly, M. J. Phys. Chem. B 2005, 109, 1691116917.

(13) Nutt, D. R; Meuwly, M. Biophys. J. 2006, 90, 1191-1201.

(14) Straub, J. E.; Karplus, M. Chem. Phys. 1991, 158, 221-248.

(15) Petrich, J. W.; Lambry, J. C.; Kuczera, K.; Karplus, M.; Poyart,

C.; Martin, J. L. Biochemistry 1991, 30, 3975-3987.

(16) Schaad, O.; Zhou, H. X.; Szabo, A.; Eaton, W. A.; Henry, E. R. Proc. Natl. Acad. Sci. U.S.A. 1993, 90, 9547-9551.

(17) Li, H.; Elber, R; Straub, J. E. J. Biol. Chem. 1993, 268, 1790817916

(18) Jensen, K. P.; Ryde, U. J. Biol. Chem. 2004, 279, 14561-14569.

(19) Dunietz, B. D.; Dreuw, A.; Head-Gordon, M. J. Phys. Chem. B 2003, 107, 5623-5629.

(20) Dreuw, A.; Dunietz, B. D.; Head-Gordon, M. J. Am. Chem. Soc. 2002, 124, 12070-12071.

(21) Harvey, J. N. J. Am. Chem. Soc. 2000, 122, 12401-12402.

(22) Frauenfelder, H.; Sligar, S. G.; Wolynes, P. G. Science 1991, 254, 1598.

(23) Gibson, Q. H.; Edelstein, J. S. J. Biol. Chem. 1987, 262, 516519.

(24) Olson, J. S.; Rohlfs, R. J.; Gibson, Q. H. J. Biol. Chem. 1987, 262, 12930-12938.

(25) Gibson, Q. H.; Regan, R.; Elber, R.; Olson, J. S.; Carver, T. E. J. Biol. Chem. 1992, 267, 22022-22034.

(26) Carlson, M. L.; Regan, R.; Elber, R.; Li, H.; Phillips, G. N.; Olson, J. S.; Gibson, Q. H. Biochemistry 1994, 33, 10597-10606.

(27) Jongeward, K. A.; Magde, D.; Taube, D. J.; Traylor, T. G. J. Biol. Chem. 1988, 263, 6027-6030.

(28) Srajer, V.; Champion, P. M. Biochemistry 1991, 30, 7390-7402.

(29) Srajer, V.; Schomacker, K.; Champion, P. Phys. Rev. Lett. 1986, $57,1267-1270$.

(30) Nienhaus, K.; Lutz, S.; Meuwly, M.; Nienhaus, G. U. Chem. Phys. Chem. 2010, 11, 119-129.

(31) Bocahut, A.; Bernad, S.; Sebban, P.; Sacquin-Mora, S. J. Phys. Chem. B 2009, 113, 16257-16267.

(32) El-Mashtoly, S. F.; Nakashima, S.; Tanaka, A.; Shimizu, T.; Kitagawa, T. J. Biol. Chem. 2008, 283, 19000-19010.

(33) Puranik, M.; Nielsen, S. B.; Youn, H.; Hvitved, A. N.; Bourassa, J. L.; Case, M. A.; Tengroth, C.; Balakrishnan, G.; Thorsteinsson, M. V.; Groves, J. T.; McLendon, G. L.; Roberts, G. P.; Olson, J. S.; Spiro, T. G. J. Biol. Chem. 2004, 279, 21906-21108.
(34) Gray, H. B.; Winkler, J. R. Chem. Phys. Lett. 2009, 483, 1-9.

(35) Wang, W.; Ye, X.; Demidov, A. A.; Rosca, F.; Sjodin, T.; Cao, W.; Sheeran, M.; Champion, P. M. J. Phys. Chem. B 2000, 104, 1078910801.

(36) Negrerie, M.; Cianetti, S.; Vos, M. H.; Martin, J. L.; Kruglik, S. G. J. Phys. Chem. B 2006, 110, 12766-12781.

(37) Zang, C.; Stevens, J. A.; Link, J. J.; Guo, L.; Wang, L.; Zhong, D. J. Am. Chem. Soc. 2009, 131, 2846-2852.

(38) Harvey, J. N. Farad. Discuss. 2004, 127, 165-177.

(39) Poli, R.; Harvey, J. N. Chem. Soc. Rev. 2003, 32, 1-8.

(40) Strickland, N.; Harvey, J. N. J. Phys. Chem. B 2007, 111, 841852.

(41) Franzen, S. J. Phys. Chem. B 2002, 106, 4533-4542.

(42) Zharikov, A. A.; Fischer, S. F. Chem. Phys. Lett. 1996, 249, 459469.

(43) Gerstman, B. S. J. Chem. Phys. 1988, 88, 6228.

(44) Gerstman, B. S.; Sungar, N. J. Chem. Phys. 1992, 96, 387.

(45) Biase, P. M. D.; Paggi, D. A.; Doctorovich, F.; Hildebrandt, P.; Estrin, D. A.; Murgida, D. H.; Marti, M. A. J. Am. Chem. Soc. 2009, 131, 16248-16256.

(46) Landau, L. Phys. Z. Sowjetunion 1932, 2, 46-51.

(47) Zener, C. Proc. R. Soc. A 1932, 137, 696-702.

(48) Frauenfelder, H.; Wolynes, P. G. Science 1985, 229, 4711.

(49) Zhu, L.; Widom, A.; Champion, P. M. J. Chem. Phys. 1997, 107, 2859.

(50) Redi, M. H.; Gerstman, B. S.; Hopfield, J. J. Biophys. J. 1981, 35, $471-484$.

(51) Jasper, A. W.; Zhu, C.; Nangia, S.; Truhlar, D. G. Faraday Discuss. 2004, 127, 1-22.

(52) Jortner, J.; Ulstrup, J. J. Am. Chem. Soc. 1979, 101, 3744.

(53) Fedorov, D. G.; Koseki, S.; Schmidt, M. W.; Gordon, M. S. Int. Rev. Phys. Chem. 2003, 22, 551-592.

(54) Minaev, B. F.; Minaeva, V. A. Ukr. Bioorg. Acta 2008, 2, 56-64.

(55) Tully, J. C. J. Chem. Phys. 1990, 93, 1061.

(56) Tully, J. C. Int. J. Quantum Chem. 1991, 25, 299-309.

(57) Rovira, C.; Carloni, P.; Parrinello, M. J. Phys. Chem. B 1999, 103, 7031-7035.

(58) Peisach, J.; Blumberg, W. E.; Wittenberg, B. A.; Wittenberg, J. B. J. Biol. Chem. 1968, 243, 1871-1880.

(59) Franzen, S.; Bohn, B.; Poyart, C.; Martin, J. L. Biochemistry 1995, 34, 1224-1237.

(60) Leu, B. M.; Ching, T. H.; Zhao, J.; Sturhahn, W.; Alp, E. E.; Sage, J. T. J. Phys. Chem. B 2009, 113, 2193-2200.

(61) Franzen, S. Proc. Natl. Acad. Sci. U.S.A. 2002, 99, 16754-16759.

(62) Petrich, J. W.; Poyart, C.; Martin, J. L. Biochemistry 1988, 27, 4049-4060.

(63) Huber, K.; Herzburg, G. Constants of Diatomic Molecules; Van Nostrand Reinhold: New York, 1979.

(64) Cianetti, S.; Negrerie, M.; Vos, M. H.; Martin, J. L.; Kruglik, S. G. J. Am. Chem. Soc. 2004, 126, 13932-13933.

(65) Rosen, N.; Zener, C. Phys. Rev. 1932, 40, 502.

(66) Kirillov, A. S. Adv. Space Res. 2004, 33, 993-997.

(67) Demkov, Y. N. Soviet Phys. JETP 1964, 18, 138.

(68) Vitanov, N. V. J. Phys. Chem. B 1994, 27, 1351-1360.

(69) Starovoitova, V.; Wyllie, G. R. A.; Scheidt, W. R.; Sturhahn, W.; Alp, E. E.; Durbin, S. M. J. Phys. Chem. B 2008, 112, 12656-12661.

(70) Autenrieth, F.; Tajkhorshid, E.; Baudry, J.; Luthey-Schulten, Z. J. Comput. Chem. 2004, 25, 1613-1622. 